\title{
O ENFRENTAMENTO DA PROBLEMÁTICA DO CÂNCER DE MAMA NA ESTRATÉGIA DE SAÚDE DA FAMÍLIA
}

\author{
Edna Rosa da Silva Nascimento ${ }^{1}$ \\ Denise Mary Costa de Oliveira ${ }^{2}$
}

RESUMO: O câncer de mama é um importante problema de saúde de pública, pois é o segundo tipo de câncer mais frequente na população e o mais comum entre as mulheres. Objetivos Identificar os problemas relacionados ao câncer de mama evidenciados na atenção primária em saúde. Método Trata-se de uma revisão integrativa. Tal revisão é um método que proporciona a síntese de conhecimento e a incorporação da aplicabilidade de resultados de estudos significativos na prática. Desenvolvimento As pacientes precisam saber sobre fatores de riscos relacionados ao Câncer de Mama que foram questionados tais como: mulheres com histórico de Câncer na família, pelo menos de um parentesco de primeiro grau, com diagnóstico de câncer de mama que tenha abaixo de 50 anos, familiar com diagnóstico de câncer de mama bilateral ou câncer de ovário, em qualquer idade, mulheres com câncer de mama masculino; com histologia de lesão mamária proliferativa com atípica ou neoplasia lobular in situ; menarca precoce; menopausa tardia, primeira gravidez após os 30 anos, multiparidade; exposição á radiação; terapia de reposição hormonal; obesidade, ingestação de álcool; sedentarismo; história familiar. Discussão Foram encontrados 12 estudos, sendo selecionados 07 para leitura dos resumos e apenas 9 publicações permanecendo para a discussão. As informações foram processadas e os resultados interpretados a partir da análise das mesmas. A pesquisa foi realizada com publicações no período de 2015 a 2020 conforme citado na metodologia. Conclusão Pode concluir que a deficiência no serviço de APS existe uma grande demanda em estar realizando os exames de mamografias, e a demora na marcação do mesmo, impede o diagnóstico precoce, para um tratamento com mais chances de cura.

Palavras-chaves: Câncer de mama. Câncer de Mama Inflamatório. Classificação Internacional de Problemas de Saúde em Atenção Primária. Primeiro Nível de Atenção.

ABSTRACT: Introduction Breast cancer is an important public health problem. as it is the second most common type of cancer in the population and the most common among women. Objectives To identify the problems related to breast cancer evidenced in primary health care. Method This is an integrative review. Such a review is a method that provides the synthesis of knowledge and the incorporation of the applicability of results of significant studies into practice. Development Patients need to know about risk factors

'Graduação de enfermagem na universisade de mogi das cruzes E-mail-ednarosa.ahm@gmail.com ${ }^{2}$ Mestre em Ciências e Tecnologia em Saúde pela Universidade de Mogi das Cruzes. Doutoranda em Engenharia Biomédica pela Universidade de Mogi das Cruzes E-mail denisemarycostanurse@gmail.com 
related to Breast Cancer that were asked, such as: women with a history of Cancer in the family, at least one first degree relationship, with a diagnosis of breast cancer who is under 50 years old, family member diagnosed with bilateral breast cancer or ovarian cancer, at any age, women with male breast cancer; with histology of proliferative breast lesion with atypical or lobular neoplasm in situ; early menarche; late menopause, first pregnancy after age 30, multiparity; radiation exposure; hormone replacement therapy; obesity, alcohol intake; sedentary lifestyle; family history. Discussion Twelve studies were found, with 07 being selected for reading the abstracts and only 9 publications remaining for discussion. The information was processed and the results interpreted from their analysis. The research was carried out with publications from 2015 to 2020 as mentioned in the methodology. Conclusion It can be concluded that the deficiency in the PHC service, there is a great demand for performing mammogram exams, and the delay in scheduling it prevents early diagnosis, for a treatment with more chances of cure.

Keywords: Breast cancer. Inflammatory Breast Cancer. International Classification of Health Problems in Primary Care. First Level of Attention.

\section{INTRODUÇÃO}

O câncer de mama é um importante problema de saúde de pública, pois é o segundo tipo de câncer mais frequente na população e o mais comum entre as mulheres. A etiologia do câncer de mama é multifatorial e envolve fatores individuais; idade, menarca precoce, menopausa tardia, ambientais; exposição á radiação; reprodutivos, primeira gravidez após os 30 anos e nuliparidades; hormonais; terapia de reposição hormonal; genéticos; história familiar; e de estilo de vida; obesidade, ingestão regular de álcool e sedentarismo (INCA 2014).

Houve uma estimativa em 2016 que haveria aproximadamente 596 mil casos novos de câncer, sendo 57.960 mil de mama feminina, constituindo a primeira causa de óbitos em mulheres, com I4 óbitos para cada ıoo mil mulheres em 2013. A taxa de mortalidade é considerada elevada por ser uma doença que o diagnóstico ainda acontece em estágios avançados (BRASIL, 2010).

O câncer de mama é uma doença causada pela multiplicação desordenada da célula que se multiplicam formando um tumor.

Há vários tipos de câncer de mama. Por isso a doença pode evoluir diferentes formas. Alguns tipos tem desenvolvimento rápido, enquanto outros crescem mais lentamente. Esses comportamentos distintos se devem a características próprias de cada tumor. $\mathrm{O}$ 
câncer de mama também acomete homens em uma porcentagem mínima, apenas I\% do total de caso da doença (INCA 2014).

O câncer de mama é o que mais acomete as mulheres em todo o mundo e também no Brasil, depois do câncer de pele não melanoma. Em 2020, são esperados 66.280 novos casos, o que representa $27 \%$ dos casos de câncer em mulher (INCA 2019). A mortalidade por essa neoplasia é ainda ascendente no país, sendo registrados em 2018, 17572 óbitos por câncer de mama e mulheres (INCA 2020).

Avanços terapêuticos nas últimas décadas vêm ampliando as possibilidades de enfrentamento da doença, porém a detecção precoce segue como desafio. A mamografia é considerada o exame padrão para rastrear o câncer de mama, mas tem limites de riscos que precisam ser conhecidos, tais como resultados falsos positivos, que causam ansiedade e necessidades de mais exames; resultados falsos negativos, que causam falsa segurança para a mulher; excesso de diagnóstico e de tratam e quando tumores que podem não evoluirsão identificados e tratados, e ainda há um pequeno risco de exposição a radiação ionizante, que pode aumentar com repetições de mamografia ao longo de muitos anos (MIGOWSKI et al., 2018 a; INCA 2015).

Considerando a necessidade de se buscar o balanço favorável entre riscos e benefícios ao rastreamento, a recomendação do ministério da Saúde é que a mamografia de rastreamento seja ofertada para as mulheres de 50 a 69 anos, a cada dois anos (INCA 2015). As mulheres devem ser informadas sobre riscos e benefícios envolvidos para que possam avaliar, participar ou não do rastreio, em um processo de decisão compartilhada (MIGOWSKI et al., 2018 b)

As diretrizes Brasileiras foram baseadas em revisões sistemáticas da literatura (MIGOWSKI et al., 2018 c) e suas recomendações de população- alvo e periocidade estão em consonância com as da Organização Mundial de Saúde (OMS) e da maioria dos países que implantam o rastreamento populacional do câncer de mama (WHO, 2014; EBELL et al., 2018).

Há, porém, outra recomendação para rastreamento monográfico no Brasil. Nela, sociedades de especialistas médicas recomendam a mamografia anual para mulheres de 40 a 74 anos, em uma diretriz não com menor rigor metodológico (URBAN et al., 2017 ;MIGOWSKI et al.,2018c). Embora essa diretriz não considere explicitamente os riscos 
mais importantes do rastreamento, é possível encontrar, de forma periférica, a menção e a necessidade de orientar as mulheres quanto aos riscos e benefícios do rastreamento em um posicionamento, em nível regional, de uma das sociedades envolvidas (VIEIRA, 2017).

A média possui papel fundamental na formação da opinião pública e na difusão de informação de saúde e recomendações para a população. Entretanto nem sempre os conteúdos veiculados possuem qualidade adequada e refletem muitas vezes interesse privados esse fato á tendência contemporânea de consumo excessivo e acrítico de tecnologia em saúde perpetua pratica que podem ser potencialmente danosas, como é o caso de rastreio mamografico em faixas etárias e periocidade não recomendada (EMERICH et al.,2016)

\section{DESENVOLVIMENTO}

O Câncer de mama hoje é considerado um problema de saúde pública. No caso de neoplasia maligna, a incidência é bem maior em mulheres na maioria parte do mundo. Nas estatísticas mundiais, foram estimados 2,I milhões de novos casos de Câncer de mama e 627 mil óbitos causados pela doença. (Brasil 2013). Os principais sintomas do Câncer de mama são observados pela paciente que são: Nódulos seguidos de dor e secreção mamária. As pacientes têm informações sobre os sinais predominantes do Câncer de mama, secreção mamilar, principalmente quando unilateral e espontânea, hiperemia na pele da mama, edema cutânea análogo á casca de laranja, retração cutânea, dor ou inversão mamilar, descamação ou ulceração do mamilo. (Brasil 2013).

As pacientes precisam saber sobre fatores de riscos relacionados ao Câncer de Mama que foram questionados tais como: mulheres com histórico de Câncer na família, pelo menos de um parentesco de primeiro grau, com diagnóstico de câncer de mama que tenha abaixo de 50 anos, familiar com diagnóstico de câncer de mama bilateral ou câncer de ovário, em qualquer idade, mulheres com câncer de mama masculino; com histologia de lesão mamária proliferativa com atípica ou neoplasia lobular in situ; menarca precoce; menopausa tardia, primeira gravidez após os 30 anos, multiparidade; exposição á radiação; terapia de reposição hormonal; obesidade, ingestação de álcool; sedentarismo; história familiar (Brasil, 2013). 
As estimativas no Brasil de índices de Câncer de Mama em 2019 foram de 59.700 novos casos, representando 29, 5\% de cânceres em mulheres, executando- se o câncer de pele não melanoma, no ano de 2016 ocorreram 16.069 mortes de mulheres por câncer de mama no Brasil.

O tratamento, o diagnóstico, o tratamento, local e o tratamento sistêmico para detectar o câncer de mama, estão sendo aprimorados de forma mais rápida, em razão do conhecimento da história natural. (Brasil 2015).

Quando umtumor invasivo é detectado é indicado um tratamento para iniciar a maior probabilidade de cura. $\mathrm{Na}$ expectativa da realização do rastreamento do câncer de mama no atendimento primário que é realizado nas UBS nota- se que na atenção secundária realizada pelo Enfermeiro, junto com o médico desempenham um papel importante na intensificação das ações. Eles são responsáveis pelas ações de rastreamento do câncer de mama dando um foco maior nas realizações de exames da mama.

È visível na assistência desses profissionais está pautada tanto na detecção precoce da doença, como é o atendimento das pacientes assintomáticas, buscando um diagnóstico completo como detectar um câncer de mama no estágio menos avançado possível, no rastreamento, que é a busca ativa do câncer. (Brasil 2017).

O Câncer de Mama pode ser diagnosticado através de ferramentas mais simples, como o exame clínico das mamas. Para realizar o exame clínico devemos ter mão de obra qualificada e treinada. O Enfermeiro na sua atuação direta deve estar atento para não somente realizar o exame clínico das mamas, mas também deve coordenar atividades na estratégia Saúde da Família, buscando sempre atingir o alvo que são as mulheres que ainda que não foram comtempladas pelo exame de mamografia. Nesse caso o enfermeiro deve também as atividades de educação relacionadas á mamografias e autoexames das mamas, assim procurando romper com preconceitos e medos que acabam se tornando barreiras no rastreamento de câncer de mama (Brasil, 2013).

O Ministério da Saúde preconiza para mulheres com faixa etária entre 40 á 49 anos, o exame clínico das mamas, anualmente. Já nas mulheres com a faixa etária entre 50 e 69 anos, é indicada a mamografia anualmente mais exame clínico das mamas. Com exceção as mulheres com 35 anos que fazem parte do grupo de risco para desenvolvimento do 
câncer de mama, devem realizar o exame clínico das mamas e a mamografia anualmente. (BARRETO ASB, Mendes MFM (citado em Mar 2017).

No Rastreamento do câncer de mama devem ser usadas ferramentas nos serviços de Saúde nas UBS na atenção primária. Todos os profissionais devem estar envolvidos no atendimento para realizar o exame clínico das mamas mesmo que o motivo seja uma consulta e não haja queixas mamárias, e orientar a paciente sobre hábitos saudáveis, autoexame das mamas cuja atribuição é proporcionar segurança em estar conhecendo o próprio corpo.

A Enfermagem apresenta em sua função do dia- a- dia vive vários tabus e
preconceitos que ocorrem desde a formação até a prática profissional. Esses tabus
estão ligados á sexualidade, que é uma "dimensão inerente ao ser humano e que
está intrinsecamente ligado aos cuidados que nós profissionais da saúde
prestamos ao paciente." (Brasil 2017).

Apesar de alguns avanços tecnológicos em vários países em desenvolvimento, o Brasil ainda possui déficit em relação á deteç̧ão precoce do Câncer de mama.

Esse déficit estende á baixa em diagnósticos e estágios da doença menos avançados, e o mais importante, e o tempo que se leva para dar início ao tratamento adequado, o que vem a dificultar um prognóstico favorável.

Se o diagnóstico for tardio e o início do tratamento, piores serão as complicações da situação da mulher, e bem como a da sua família. (Brasil 2017).

O SUS não oferece suporte para realizar exames de mamografia á curto prazo, por isso há a possibilidade do avanço de câncer de mama, mau prognóstico e os sofrimentos".

No SUS Sistema único de Saúde houve um grande debate sobre as limitações em termos de cobertura monográficas, e sobre as dificuldades, no controle e avaliação dos serviços prestados. Enquanto há um número de mamografias duas vezes maior supera a quantidade para suprir a população, porém são distribuídos e com baixo nível de produtividade. Alguns fatores contribuem para essa deficiência como fatores lucrativos. (Brasil, 2017).

Atualmente são atendidos pelo SUS/SP mais de 722 mil de mamografias / ano. A quantidade de equipamentos de equipamentos no SUS não supera a quantidade necessária para realização de mamografias seguindo critérios da Portaria MS n 1631/2015, que no caso de mamógrafos que realizam uma produção potencial de 3,6 milhões de exames. Em 2018 
foram totalizando a produção de mamógrafos I,4 milhões para rastreamento e outros tipos de indicação. Assim foi possível ampliar o acesso mais rápido para realização de mamografias e nos serviços existentes (Brasil, 2008).

Existem, porém algumas situações, locais com menor acesso pela distância e dificuldades de locomoção aos serviços localizados em centros das cidades, que poderão ser atendidas por unidades móveis de mamografias. A importância da divulgação da realização das mamografias tem como atuante sobre o pessoal técnico do setor de saúde, as entidades da sociedade Civil, os gestores do SUS e a própria população por meio de campanhas com informações em todo o Estado de São Paulo, imprescindíveis para melhorar as realizações do exame, e garantir o tratamento adequado, além da promoção, prevenção de saúde, orientação para alimentação adequada, atividade física e outros fatores que estão interligados á redução de risco do câncer de mama, podendo beneficiar todas as mulheres. (INCA, 2015).

\section{MÉTODO}

\section{TIPO DE PESQUISA}

Trata-se de uma revisão integrativa. Tal revisão é um método que proporciona a síntese de conhecimento e a incorporação da aplicabilidade de resultados de estudos significativos na prática (SOUZA et al 2016).

\section{LOCAL DE PESQUISA E DESCRITORES}

A busca dos dados do presente estudo será realizada por meio de uma consulta informatizada no banco de dados bibliográficos da Biblioteca Virtual em Saúde (BVS) com artigos indexados nas bases de dados da Literatura Latino-Americana em Ciências de Saúde (LILACS), e Scientific Eletronic Library Online (SCIELO), por apresentarem expressivo acervo na temática desse estudo.

Os descritores utilizados nessa pesquisa serão: Enfermagem ,câncer de mama Rastreamento, mamografia, Enfretamento, Atenção Primária. Validados pelos Descritores de Ciência em Saúde (DECs). 


\section{CRITÉRIOS DE INCLUSÃO E EXCLUSÃO}

$\mathrm{Na}$ busca de resultados, serão considerados os seguintes critérios de inclusão: Artigos científicos que retratam sobre o tema e os descritores expostos.

Serão considerados critérios de exclusão: Publicações anteriores a 2010, artigos na língua estrangeira e, que não contemplam a temática.

\section{TÉCNICA DE COLETA DOS DADOS E ANÁLISE DE CONTEÚDO}

Para a busca de títulos e resumos afins, será selecionado o descritor principal "recém-nascido" sendo cruzados com cada um dos demais descritores do estudo.

Após o levantamento da literatura relacionada à temática, será realizada seleções de textos pertinentes com a categorização e interpretação das ações não farmacológicas implementadas pela Enfermagem a mulheres, segundo a análise de conteúdo (BARDIN, 2004).

Para Bardin (2004) inicialmente faz-se uma criteriosa leitura dos artigos para o agrupamento de informações de significados semelhantes, levando à formação de subtemas, elaborados pelo pesquisador, podendo ser organizados em forma de Quadros contendo: nome dos autores, título do estudo, objetivos e respectivos achados, sendo analisados na discussão do estudo.

Esse projeto não necessitará ser submetido ao Comitê de Ética da Universidade de Mogi das Cruzes, por tratar-se de revisão integrativa, segundo os preceitos da Resolução do Conselho Nacional de Saúde 466/ı2.

\section{RESULTADOS E DISCUSSÃO}

Foram encontrados 12 estudos, sendo selecionados 07 para leitura dos resumos e apenas 9 publicações permanecendo para a discussão. As informações foram processadas e os resultados interpretados a partir da análise das mesmas. A pesquisa foi realizada com publicações no período de 2015 a 2020 conforme citado na metodologia. Os artigos selecionados para a pesquisa foram avaliados através da leitura dos títulos que condiziam com o tema estudado. Em seguida, foram avaliados os resumos que continham 
informações referentes ao tema abordado, e por fim, a leitura completa dos artigos que apresentam o conteúdo para atender o objetivo proposto.

Os dados foram apresentados em quadros possibilitando uma melhor visualização dos dados levantados na leitura e interpretação científica, seguidos de sua discussão. Segue abaixo o quadro das principais características dos estudos utilizados na pesquisa.

Quadro I: Caracterização dos problemas evidenciados no câncer de mama na atenção primária.

\begin{tabular}{|c|c|c|c|}
\hline AUTORES ANO & MÉTODO & $\begin{array}{c}\text { PALAVRAS } \\
\text { CHAVES }\end{array}$ & ACHADOS \\
\hline $\begin{array}{l}\text { Ministério } \\
\text { Saúde, } \\
\text { Migowshi et. al, } \\
2018\end{array}$ & $\begin{array}{l}\text { Pesquisa } \\
\text { com } \\
\text { abordagem } \\
\text { qualitativa }\end{array}$ & $\begin{array}{l}\text { Câncer de } \\
\text { mama, } \\
\text { conhecimentos e } \\
\text { práticas dos } \\
\text { Enfermeiros de } \\
\text { uma APS. }\end{array}$ & $\begin{array}{l}\text { As principais dificuldades dos } \\
\text { Enfermeiros no rastreamento } \\
\text { relacionados ao tratamento e } \\
\text { prevenção sobre o câncer } \\
\text { demama associam-se a mulheres } \\
\text { de faixa etária de 4I a } 59 \text { anos. }\end{array}$ \\
\hline INCA, 2020 & $\begin{array}{l}\text { Revisão } \\
\text { Bibliográfic } \\
\text { a }\end{array}$ & $\begin{array}{l}\text { Câncer de } \\
\text { mama, tipos, } \\
\text { desenvolvimento }\end{array}$ & $\begin{array}{l}\text { No mundo inteiro as mulheres } \\
\text { são mais acometidas pelo câncer } \\
\text { de Mama. Em } 2020 \text { foram } \\
\text { esperados o numero de mulheres } \\
\text { com câncer de mama } 66.280 . \\
\text { Nesses casos podemos afirmar } \\
\text { que } 27 \% \text { de casos em mulheres } \\
\text { no Brasil. Algum tipo de câncer } \\
\text { se desenvolve muito mais } \\
\text { rápido, enquanto outros crescem } \\
\text { lentamente. }\end{array}$ \\
\hline Inca, 2018 & $\begin{array}{l}\text { Pesquisa } \\
\text { com } \\
\text { Revisão }\end{array}$ & $\begin{array}{l}\text { Promoção á } \\
\text { saúde, Atenção } \\
\text { primária, }\end{array}$ & $\begin{array}{l}\begin{array}{l}\text { Detecção precoce do Câncer, } \\
\text { diagnóstico } \\
\text { rastreamento do Câncer, estagio }\end{array}\end{array}$ \\
\hline
\end{tabular}




\begin{tabular}{|c|c|c|c|}
\hline & $\begin{array}{l}\text { bibliográfic } \\
\text { a }\end{array}$ & $\begin{array}{l}\text { Neoplasias de } \\
\text { mama. }\end{array}$ & $\begin{array}{l}\text { avançado da doença, } \\
\text { mortalidade, morbidade. }\end{array}$ \\
\hline Vieira, 2017 & $\begin{array}{l}\text { Pesquisa } \\
\text { com } \\
\text { Revisão } \\
\text { bibliográfic } \\
\text { a }\end{array}$ & $\begin{array}{l}\text { Rastreamento de } \\
\text { câncer de mama } \\
\text { nos serviços de } \\
\text { APS }\end{array}$ & $\begin{array}{l}\text { Primeiros Sinais que podem } \\
\text { evidenciar o Câncer de mama. } \\
\text { Realização do Autoexame. } \\
\text { Demora na marcação da } \\
\text { mamografia, pois há uma grande } \\
\text { demanda e poucos Mamógrafos. }\end{array}$ \\
\hline Brasil, 2017 & $\begin{array}{l}\text { Revisão } \\
\text { Bibliográfic } \\
\text { a }\end{array}$ & $\begin{array}{l}\text { Conhecimento } \\
\text { das mulheres } \\
\text { sobre os exames } \\
\text { que os } r \text { serviços } \\
\text { de } \\
\text { oferecem. }\end{array}$ & $\begin{array}{l}\text { Os exames que a APS oferece } \\
\text { são: Autoexame, Ultrassom das } \\
\text { mamas, Mamografias. }\end{array}$ \\
\hline Inca 2015 & $\begin{array}{l}\text { Revisão } \\
\text { Bibliográfic } \\
\text { a }\end{array}$ & $\begin{array}{ll}\text { Riscos } & e \\
\text { benefícios } & \text { da } \\
\text { mamografia no } & \\
\text { rastreamento } & \end{array}$ & $\begin{array}{l}\text { Avaliar, participar ou não do } \\
\text { rastreio. } \\
\text { Implantar o rastreamento na } \\
\text { população em geral. } \\
\text { Oferecer, as realizações de } \\
\text { mamografia entre mulheres com } \\
\text { faixa etária entre 5o a } 69 \text { anos a } \\
\text { cada dois anos. }\end{array}$ \\
\hline $\begin{array}{l}\text { Migowshi, } \\
2018\end{array}$ & $\begin{array}{l}\text { Revisão } \\
\text { Bibliográfic } \\
\text { a }\end{array}$ & $\begin{array}{l}\text { Exames } \\
\text { recomendado } \\
\text { pela OMS e o } \\
\text { rastreamento da } \\
\text { população com } \\
\text { Câncer de Mama }\end{array}$ & $\begin{array}{l}\text { O rastreamento é recomendado a } \\
\text { mamografia ser realizada uma } \\
\text { vez por ano. A OMS também } \\
\text { preconiza que seja realizada a } \\
\text { mamografia anual entre } \\
\text { mulheres na faixa etária de } 40 \text { a }\end{array}$ \\
\hline
\end{tabular}




\begin{tabular}{|c|c|c|c|}
\hline & & & 74 anos. \\
\hline $\begin{array}{l}\text { Instituto Nacional } \\
\text { de Câncer José } \\
\text { Alencar Gomes da } \\
\text { Silva; INCA } 2015 \text {. }\end{array}$ & $\begin{array}{l}\text { Revisão } \\
\text { Bibliográfic } \\
\text { a }\end{array}$ & $\begin{array}{l}\text { Promoção á } \\
\text { saúde, Prevenção } \\
\text { á saúde, na } \\
\text { redução de riscos } \\
\text { do Câncer de } \\
\text { Mama. }\end{array}$ & $\begin{array}{l}\text { A importância de estar } \\
\text { realizando anualmente as } \\
\text { mamografias. } \\
\text { Tratamento adequado. } \\
\text { Atividades físicas e outros } \\
\text { fatores que estão ligados á } \\
\text { redução de risco de Câncer. }\end{array}$ \\
\hline $\begin{array}{l}\text { EMERICK et.al., } \\
2016\end{array}$ & $\begin{array}{l}\text { Revisão } \\
\text { Bibliográfic } \\
\text { a }\end{array}$ & $\begin{array}{l}\text { O rastreamento } \\
\text { vinculado á } \\
\text { tecnologia. }\end{array}$ & $\begin{array}{l}\text { O avanço da tecnologia podem } \\
\text { torna-se as práticas que podem } \\
\text { se tornar donosas, e no caso do } \\
\text { rastreamento mamógrafico em } \\
\text { faixa etárias e periodicamente } \\
\text { não recomendada. }\end{array}$ \\
\hline
\end{tabular}

Fonte: Elaborado pela própria autora, 2021.

Nesse estudo podemos constatar que a maioria das mulheres que utilizam o serviço de APS para rastreamento de câncer de mama, são mulheres que tem ensino fundamental completo $15 \%$, ensino fundamental incompleto $15 \%$, ensino médio completo $10 \%$, ensino médio incompleto 20\%, e mulheres sem instrução de escolaridade $25 \%$.

Podemos relatar que esses grupos subdivididos conforme o grau de escolaridade, que nem todas as mulheres têm conhecimentos de algumas informações sobre ações e tipos de prevenções sobre o câncer de mama, pois os serviços de saúde por sua vez acabam tendo uma demanda muito grande, e a demora de realização de exames de mamografias. Dificultando assim o diagnóstico precoce do câncer, para iniciar o tratamento, e prolongar vida dessas mulheres no caso de diagnóstico tardio.

Quadro 2: Enfrentamento de problemas durante o câncer de mama. 


\begin{tabular}{|c|c|c|}
\hline AUTOR/ ANO & TITULO & PRINCIPAIS ATUAÇÕES \\
\hline $\begin{array}{c}\text { INCA } \\
2013\end{array}$ & $\begin{array}{l}\text { Proposta de ações do Enfermeiro na APS na } \\
\text { detecção precoce do Câncer de Mama }\end{array}$ & $\begin{array}{l}\text { Conhecimentos, habilidades, } \\
\text { reconhecer e definir condutas. } \\
\text { Criar ações, coordenar } \\
\text { atividades na Estratégia Saúde } \\
\text { da Família, buscando alcançar } \\
\text { o público alvo que são as } \\
\text { mulheres que ainda não } \\
\text { conseguiram realizar a } \\
\text { mamografia. } \\
\text {. }\end{array}$ \\
\hline BRASIL,2017 & $\begin{array}{l}\text { SUS um sistema com dificuldades para realizar } \\
\text { mamografias. }\end{array}$ & $\begin{array}{l}\text { Demanda muito grande em } \\
\text { realizar a necessidade da } \\
\text { população que precisa realizar } \\
\text { a mamografia, e são } \\
\text { distribuídos com baixo nível } \\
\text { de produtividade. }\end{array}$ \\
\hline BRASIL , 2015 & Estimativas do Câncer de Mama no Brasil & $\begin{array}{l}\text { Estima-se que } 2019 \text { o número } \\
\text { de câncer de mama foram de } \\
59.700 \text { novos casos Em } 2020 \\
\text { foram registrado mais de } \\
66.280 \text { casos de câncer de } \\
\text { mama. }\end{array}$ \\
\hline
\end{tabular}

Fonte: Elaborado pela própria autora, 2021.

Em muitas vezes sem condições os profissionais que trabalham na APS se vem em uma situação difícil, e muitas vezes se sentem impotentes de estar dando um auxilio, ouuma assistência adequada, e de direcionar essas mulheres para um tratamento rápido, já que o sistema do serviço prestado na APS tem suas limitações e dificuldades para iniciar o tratamento, ou realizações de exames imediatos.

Infelizmente o SUS tem uma demanda muito grande de exames a serem realizados, e poucos mamógrafos para atender metade da população que necessitam de realizar esses exames com certa urgência.

Recentemente os serviços de saúde conveniados ao SUS, através da parceria com o Ministério da Saúde, tem observado a importância de compras de equipamentos de mamografia ( os mamógrafos), para detecção precoce do câncer de mama muito mais rápido, com maior eficácia, e eficiência no rastreamento, diminuindo o número de casos, e proporcionando qualidade de vida a essas mulheres. 


\section{CONCLUSÃO}

Podemos concluir que a deficiência no serviço de APS existe uma grande demanda em estar realizando os exames de mamografias, e a demora na marcação do mesmo, impede o diagnóstico precoce, para um tratamento com mais chances de cura.

Também podemos citar á deficiência de equipamentos de mamografias (os mamógrafos), dificultando a rapidez de realizações dos exames, diminuindo o número de casos, realizando assim com eficácia no diagnóstico e tratamento.

O SUS com toda a deficiência no atendimento não somente em mulheres com câncer, em todo atendimento assistencial tem dado uma grande importância nas compras de mais mamógrafos para á população que moram em lugares de difícil acesso, que não tem acesso a região central, dando a chance de realizar os exames de uma maneira mais rápida e segura.

\section{REFERÊNCIAS}

Brasil. Secretária de Atenção á Saúde. Departamento de atenção Básica. Caderno de Atenção Básica. Rastreamento.v.29. Brasília: ministério da Saúde. 2015 BRASIL. Ministério da Saúde. Secretaria de Vigilância á Saúde. Departamento de Análises de Situação de Saúde. Plano de ações Estratégicas para o enfrentamento das doenças Crônicas não transmissíveis (DCNT) no Brasil. Brasília: Ministério da Saúde; 2011.

BRASIL, Instituto Nacional de Câncer. Parâmetros Técnicos para Programação de Açõesde Detecção Precoce do Câncer da Mama - Recomendações para Gestores Estaduais e Municipais. Rio de Janeiro: INCA, 2006.

CASTRO, Elisa KernKern de; LAWRENZ, Priscila; ROMEIRO, Fernanda; LIMA, Natália Britz de. HAAS, Sílvia Abduch. Percepção da Doença e Enfrentamento em Mulheres com Câncer de Mama. Psicologia: Teoria e Pesquisa. Vol. 32 n. 3, pp. I-6, Jul-Set 2016

INCA. ABC do câncer: abordagens básicas para o controle do câncer / Instituto Nacional de Câncer José Alencar Gomes da Silva; organização Mario Jorge Sobreira da Silva. - 4. ed. rev. atual. - Rio de Janeiro: Inca, 2018.

MIGOWSKI,A. et al. Diretrizes para detecção precoce do Câncer da Mama no Brasil.III- Desafios á implementação. Caderno de Saúde pública, Rio de Janeiro. 34, n. 6 e 0.046. 317, 2018 b.

MIGOWSKI, A. et al. Diretrizes para detecção precoce do câncer da mama no Brasil. I. Métodos e Elaboração. Caderno de saúde pública, Rio de Janeiro, v. 34, n 6 e oo63. 17, 2018 c 
VIEIRA, SC. Câncer de mama: Consenso da Sociedade Brasileira de MastologiaRegional Piauí. Teresina: EDUFPI, 2017.

YAMAGUCHI ,N.H. O Câncer na Visão da Oncologia. In CARVALHO, M.M.M.J. (coord.). Introdução á psiconcologia. Campinas: Livro Pleno, 2002. Cap.I, p. 21- 32. 